\title{
French users need European neutrons
}

\section{Big projects may not be popular at present, but policy-makers must think of the future.}

Sir - As pointed out in your Opinion article "Anyone for neutrons?" (Nature 417, 883; 2002), the European Spallation Source (ESS) user meeting in May was an impressive demonstration of the lively activity of European neutron users. Five propositions for hosting the site of the ESS were presented, with backing from regional and/or national authorities.

It was surprising and regrettable to note the absence of a proposal for a French site. France has a long and deep tradition in the field of scientific use of neutron beams. It hosts the most powerful neutron source in the world, the Institut Laue Langevin (Grenoble), and a modern and wellequipped national source, the Laboratoire Léon Brillouin (Saclay). The French user community has declared its scientific interest in the ESS project on several occasions, and the French Neutron Users Society (www.sfn.asso.fr) has fully approved the goal of a new-generation neutron source in the next decade. A French team is heavily involved in the design and evaluation of the ESS.

Despite this commitment, no French site was proposed at Bonn, reflecting the obstacles raised by the diverging approaches of its national bodies (the Ministry of Research and Technology, and the two major funding agencies, the CEA and the CNRS). Moreover, the absence of high-level decision-makers representing French authorities at Bonn appeared as a sign of indifference.

It may be difficult in France at the moment to support large research infrastructures, and the financial climate may be unfavourable. But this does not excuse sacrificing the future. One cannot make progress by sticking to fixed schemes of funding and maintaining a conservative outlook on the merits of neutron science.
The Bonn meeting showed the multidisciplinary and scientific potential of the ESS project. The present sources are needed to assure continuity, and to maintain and complete the training of the scientists who will run and use the source when it is completed.

As is usual in the case of any largefacility project, the construction phase of the ESS would require funding beyond the level of current functioning. But, considering the rapid expansion of the areas of science that will benefit from the new facility, the running costs will surely be reasonable.

F. Leclercq (president) ${ }^{\star}$, H. Mutka (vicepresident) $\dagger$, Société Française de la Neutronique

${ }^{\star}$ LASIR UMR 8516 du CNRS, 13 rue de Toul, F-59046, Lille Cedex, France

$\dagger$ Institut Laue Langevin, BP 156, F-38042

Grenoble Cedex 9, France

\section{Shared knowledge can combat malaria}

Sir-Andrew Clark in his excellent News and Views article "Malaria variorum" (Nature 418, 283-285; 2002) discusses the extent of genetic variability exhibited in populations of Plasmodium falciparum, the causative agent of the most lethal form of malaria, and the implications for vaccine development and for the emergence of drug resistance. He concludes that a shared resource of $P$. falciparum isolates is needed to address the problem and to advance our understanding of the evolution of this species. We agree.

The Malaria Research and Reference Reagent Resource Center (MR4) was established in 1998, partly to provide the resources available to malaria researchers worldwide that Clark discusses, and is managed by the American Type Culture Collection (ATCC). Under contract to the National Institute of Allergy and Infectious Diseases (NIAID), MR4 makes reagents available free of charge to registered individuals worldwide (see www.malaria. mr4.org). Further information can be found at the NIAID website (www.niaid. nih.gov/dmid/malaria/malrep/default.htm). MR 4 currently has more than 450 reagents, including numerous isolates, strains and species of Plasmodium and Anopheles mosquitoes as well as antibodies, antigens, DNA libraries and primers and microarray chips.
Training programmes, workshops and technology transfer to malaria-endemic regions are also important components of MR4. We encourage researchers to collaborate with MR4 to develop and support the needs of the community, as well as to contribute resources that can thereby be made more widely available. Yimin Wu*, M. John Rogers $\dagger$ ${ }^{*}$ MR4/Protistology, ATCC, 10801 University Boulevard, Manassas, Virginia 20110, USA $\dagger$ National Institute of Allergy and Infectious Diseases, 6610 Rockledge Drive, Bethesda, Maryland 20892, USA

\section{Prokaryote taxonomy online: challenges ahead}

Sir — Following calls for online taxonomic databases encompassing all organisms living on Earth $^{1-4}$, we wish to point out that extensive taxonomic information on the prokaryotes is already available online. The Approved Lists of Bacterial Names, including names and their nomenclatural history, are on a website that is updated bimonthly (www.bacterio.cict.fr). On 9 July 2002 the lists named 5,866 species, classified in 1,104 genera, Archaea and Bacteria combined. Comprehensive information on prokaryote taxonomy is in two handbooks, The Prokaryotes and Bergey's Manual of Systematic Bacteriology, both published by Springer-Verlag. The former is published online

(www.link.springer.de/link/service/books/
10125), and the database of the second edition of the latter (www.cme.msu.edu/ bergeys) exists in electronic form, so could easily be made available on the web if the publisher gives permission.

In the case of the prokaryotes, obstacles virtually unknown to the botanist and the zoologist must be overcome to achieve the goals mentioned by Gewin and others. First, the species concept for bacteria is still not well-defined ${ }^{5,6}$. Second, sequencing of $16 \mathrm{~S}$ ribosomal RNA genes collected from terrestrial and aquatic environments shows that the number of known prokaryotic species probably forms only a fraction of $1 \%$ of the number in nature. Morphological diversity is small, and therefore digital images of type specimens are of little help in online taxonomic databases. The lack of innovative culturing techniques and of well-trained taxonomists to study the isolates are of great concern. Some of the most abundant prokaryotes on Earth defy microbiologists' attempts to isolate them. Here lies one of the major challenges to future taxonomists.

\section{Aharon Oren ${ }^{\star}$, Erko Stackebrandt $\dagger$}

${ }^{\star}$ Institute of Life Sciences and Moshe Shilo Minerva Center for Marine Biogeochemistry, The Hebrew University of Jerusalem, 91904 Jerusalem, Israel $\dagger$ Deutsche Sammlung von Mikroorganismen und Zellkulturen GmbH, Mascheroderweg $1 b$,

D-38124 Braunschweig, Germany

1. Godfray, H. C. J. Nature 417, 17-19 (2002).

2. Lee, M. S. Y. Nature 417, 787-788 (2002).

3. Gewin, V. Nature 418, 362-363 (2002).

4. Bisby, F. A. et al. Nature 418, 367 (2002).

5. Cohan, F. M. Syst. Biol. 50, 513-524 (2001).

6. Stackebrandt, E. et al. Int. J. Syst. Evol. Microbiol. 52, 1043-1047 (2002). 